\title{
Optimal Robot Path Selection Using Fuzzy Analytical Hierarchical Process ${ }^{+}$
}

\author{
Abhijeet Ravankar*, Ankit A. Ravankar, Yukinori Kobayashi and Takanori Emaru \\ Lab of Robotics and Dynamics, Graduate School of Engineering, Hokkaido University, Sapporo 060-8628, Japan; \\ ankitravankar@gmail.com (A.A.R.); kobay@eng.hokudai.ac.jp (Y.K.); emaru@eng.hokudai.ac.jp (T.E.) \\ * Correspondence: abhijeetravankar@gmail.com \\ + Presented at the 4th International Electronic Conference on Sensors and Applications, 15-30 November 2017; \\ Available online: http:/ / sciforum.net/conference/ecsa-4.
}

Published: 14 November 2017

\begin{abstract}
Mobile robots are faced with problems (for ex. path planning) with many alternative solutions (ex. paths) based on several factors, and they must make a selection by quantifying the factors and mathematically evaluating the alternative solutions. Robot path planning is an integral process of mobile robots. A shortest path is generally chosen, however, it is not necessarily the optimal path. Apart from the distance between the start and goal locations, a robot must consider several other factors like the bumpiness, steepness, and crowd on the path. Robots are equipped with sensors like cameras, inertial sensors, and distance sensors to measure these factors. Different paths could be generated between the same start and goal locations considering these factors. The robot must select the optimal path from many paths. The factors which influence the generation of such paths can be dynamic. In this paper we propose to use Fuzzy Analytical Hierarchical Process (Fuzzy-AHP) to analytically select the optimal path from different paths. Fuzzy-AHP provides two navigational approaches, namely, defensive and offensive approaches which can be taken by mobile robots for navigation. In this paper, we present a case study of robot path selection with Fuzzy-AHP.
\end{abstract}

Keywords: robot path planning; Analytical Hierarchical Process; optimal robot path; robot sensors

\section{Introduction}

A mobile robot continuously makes decisions: for ex. to stop or turn to avoid collision, to increase or decrease speed, or to make way for other people. For navigation to service locations, it needs to plan a path from its current location to the goal location. Many algorithms like A-star, D-star, Probabilistic Roadmap, Rapidly-exploring random tree, Dijkstra, and other planners have successfully been used for path planning [1]. Most of these planners generate a shortest path from the start location to the goal location. However, the shortest path is not necessarily the best (for ex. safest) path. Apart from the distance between the start and goal locations, a robot must consider several other factors like the bumpiness (i.e., terrain roughness), steepness, and crowd [2] on the path.

This is shown in Figure 1 where a robot must select a path from start to goal locations considering several factors. Depending on the sensors used by the robots, other factors like the brightness of the path, for ex. in case of vision sensors, must also be considered. Robots are equipped with sensors like cameras, inertial sensors, and distance sensors to measure these factors. For example, inertial sensor like IMU is used to map the roughness of the terrain. Stereo cameras are used to detect people on the path and estimate the crowd. RGBD sensors (ex. Microsoft Kinect) can be used to estimate the steepness of the path. Generally, a map [3] is available to the robot in which areas like bumpy road, and steep paths can be marked beforehand. Factors like crowd on the path can be estimated in real-time. There could be different paths between the same start and goal locations, and the robot must select the optimal path from many paths. The factors which influence the generation of such paths can 
be dynamic. Moreover, each factor has a varying degree of influence on the selection of path by the robot, which too varies from robot to robot.

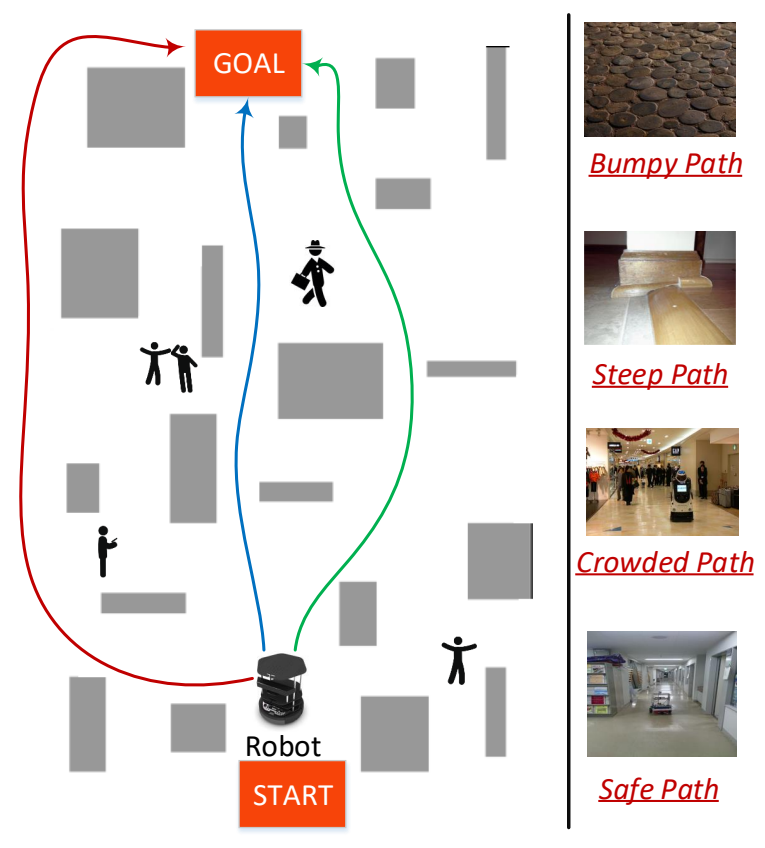

Figure 1. Selecting an optimal path from different paths based on several parameters.

In such situations, selecting a particular path based on a rigorous mathematical analysis which validates the particular selection becomes necessary. We show that Fuzzy Analytical Hierarchical Process (Fuzzy-AHP) can be used in such situations to make the optimal selection of the path considering multiple factors. AHP was originally proposed by Saaty [4] and has been employed widely in multiple criteria decision making problems involving robots [5-7]. AHP has extensively been explained in the work of Alam et al. [8]. We demonstrate the process step-by-step by taking a case study of path selection.

\section{Brief Explanation of AHP}

Readers may find the detailed explanation of AHP in $[4,8,9]$. A very brief overview is presented here, and AHP will be explained by taking a concrete use case. There are three components of AHP: (1) Hierarchy construction; (2) Priority analysis; and (3) Consistency verification. For a given problem, if there are $n$ factors $F_{1}, F_{2}, \cdots, F_{n}$ and their weights (importance) are indicated as $w_{1}, w_{2}, \cdots, w_{n}$, the pairwise comparison of evaluation factors can be represented as a matrix:

$A=\left(\begin{array}{c|cccc|cc} & F_{1} & F_{2} & \cdots & F_{n} & \text { Geometric Mean } & \text { Normalized Weight } \\ \hline F_{1} & \frac{w_{1}}{w_{1}} & \frac{w_{1}}{w_{2}} & \cdots & \frac{w_{1}}{w_{n}} & \sqrt[n]{\prod_{i=1}^{n}\left(\frac{w_{1}}{w_{i}}\right)} & \sqrt[n]{\prod_{i=1}^{n}\left(\frac{w_{1}}{w_{i}}\right)} / \Theta \\ F_{2} & \frac{w_{2}}{w_{1}} & \frac{w_{2}}{w_{2}} & \cdots & \frac{w_{2}}{w_{n}} & \sqrt[n]{\prod_{i=1}^{n}\left(\frac{w_{2}}{w_{i}}\right)} & \sqrt[n]{\prod_{i=1}^{n}\left(\frac{w_{2}}{w_{i}}\right)} / \Theta \\ \vdots & \vdots & \vdots & \vdots & \vdots & \vdots \\ F_{n} & \frac{w_{n}}{w_{1}} & \frac{w_{n}}{w_{2}} & \cdots & \frac{w_{n}}{w_{n}} & \sqrt[n]{\prod_{i=1}^{n}\left(\frac{w_{n}}{w_{i}}\right)} & \sqrt[n]{\prod_{i=1}^{n}\left(\frac{w_{n}}{w_{i}}\right)} / \Theta\end{array}\right), \Theta=\sum_{k=1}^{n} \sqrt[n]{\prod_{i=1}^{n}\left(\frac{w_{k}}{w_{i}}\right)}$. 
In matrix $\mathrm{A}, A_{i j}=\frac{1}{A_{j i}}$ for the factors. In AHP, the pairwise comparison matrices for each of the factors $F_{i}, i \in n$ is determined individually. Later, the overall evaluation of alternatives is determined. Thus, in AHP once the hierarchy is built, the various elements are compared to each other in terms of their impact with other elements in the hierarchy [10]. This comparison often involves human judgments in performing the evaluations.

\section{Use Case: Applying AHP for Path Planning}

We now consider a concrete case of optimal path selection by robot. It is assumed that the robot has the map of the environment $[3,11]$ in which the obstacles and open areas have been marked. We first start with the hierarchy chart shown in Figure 2. The hierarchy chart shows the topic: 'Path Planning from Location $X$ to $Y^{\prime}$. There are 4 evaluation factors: (1) Safety of path; (2) Steepness of path; (3) Traffic on path, and (4) Bumpiness or roughness of the path. Based on these factors, it is assumed that the robot has three path alternatives: (1) Path A; (2) Path B; and (3) Path C. These paths can be generated by using any of the path planning algorithms [1,12]. The goal is to find the best path.

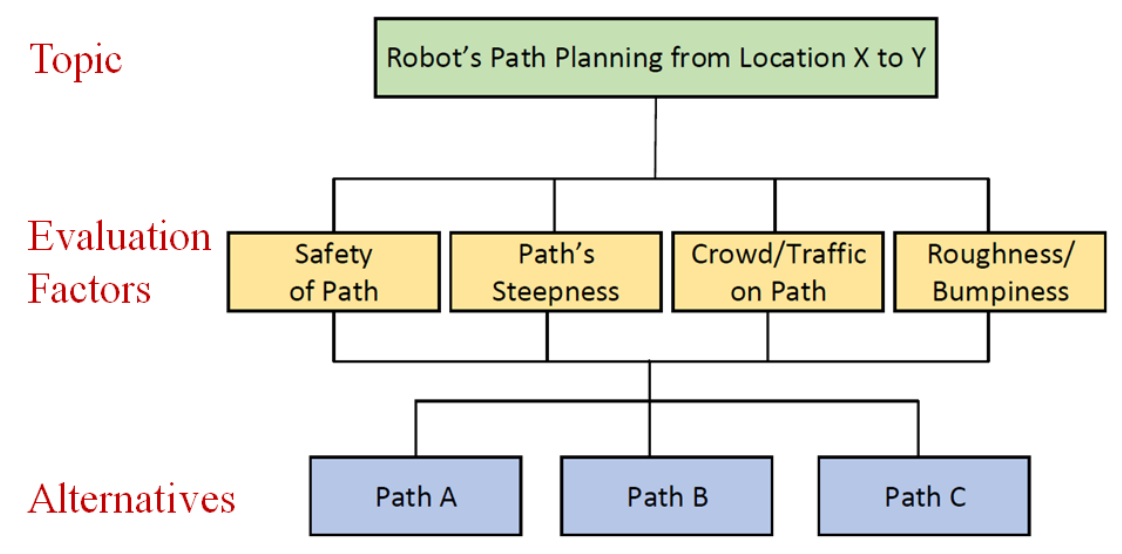

Figure 2. Hierarchy Chart.

A score is given corresponding to each degree of importance. As an example, five different scores are allotted as shown in Table 1.

Table 1. Degree of Importance and corresponding Scores.

\begin{tabular}{cc}
\hline Degree of Importance & Score \\
\hline Same Importance & 1 \\
Little More Important & 3 \\
Very Important & 5 \\
Extremely Important & 7 \\
Absolutely Important & 9 \\
\hline
\end{tabular}

Table 2 shows the comparison of evaluation factors. This is done by a human and values are manually stored in the robot. In this use case, it is assumed that 'safety' has the highest priority and the robot has difficulty to navigate over a steep terrain. Therefore, in Table 2, the element corresponding to Safety-Steepness is 9, as safety is 'absolutely important' when compared to steepness. Similarly, the other elements of the matrix are filled. A geometric mean is calculated by taking the $n$th root of the product of scores, as explained in Section 2. Later, the weights are normalized. The 'Accountable Degree' is calculated by dividing each element of the normalized weights by the maximum normalized weight, and is marked in gray color in Table 2. 
Table 2. Comparison of Evaluation Factors.

\begin{tabular}{cccccccc}
\hline & Safety & Steepness & Crowd & Bumpiness & Geometric Mean & Normalized Weight & Accountable Degree \\
\hline Safety & 1 & 9 & 3 & 5 & 3.408 & 0.581 & 1.000 \\
Steepness & $1 / 9$ & 1 & $1 / 5$ & $1 / 3$ & 0.293 & 0.050 & 0.086 \\
Crowd & $1 / 3$ & 5 & 1 & 3 & 1.495 & 0.255 & 0.436 \\
Bumpiness & $1 / 5$ & 3 & $1 / 3$ & 1 & 0.668 & 0.114 & 0.196 \\
\hline
\end{tabular}

The next step involves weight evaluations for each of the individual factors. Since there are 4 factors, the weight evaluations for safety, steepness, crowd, and bumpiness are given in Tables 3-6, respectively. The normalized weights of each evaluation is shown in Table 7 in different colors.

Table 3. Weight Evaluation for 'Safety'.

\begin{tabular}{lccccc}
\hline & Path A & Path B & Path C & Geometric Mean & Normalized Weight \\
\hline Path A & 1 & 3 & 3 & 2.080 & 0.552 \\
Path B & $1 / 3$ & 1 & $1 / 7$ & 0.362 & 0.096 \\
Path C & $1 / 3$ & 7 & 1 & 1.326 & 0.352 \\
\hline
\end{tabular}

Table 4. Weight Evaluation for 'Steepness'.

\begin{tabular}{lccccc}
\hline & Path A & Path B & Path C & Geometric Mean & Normalized Weight \\
\hline Path A & 1 & $1 / 5$ & $1 / 7$ & 0.306 & 0.072 \\
Path B & 5 & 1 & $1 / 3$ & 1.186 & 0.279 \\
Path C & 7 & 3 & 1 & 2.759 & 0.649 \\
\hline
\end{tabular}

Table 5. Weight Evaluation for 'Crowd'.

\begin{tabular}{lccccc}
\hline & Path A & Path B & Path C & Geometric Mean & Normalized Weight \\
\hline Path A & 1 & 5 & $1 / 3$ & 0.405 & 0.110 \\
Path B & $1 / 5$ & 1 & $1 / 9$ & 0.281 & 0.076 \\
Path C & 3 & 9 & 1 & 3.000 & 0.814 \\
\hline
\end{tabular}

Table 6. Weight Evaluation for 'Bumpiness'.

\begin{tabular}{lccccc}
\hline & Path A & Path B & Path C & Geometric Mean & Normalized Weight \\
\hline Path A & 1 & $1 / 9$ & $1 / 5$ & 0.281 & 0.063 \\
Path B & 9 & 1 & 3 & 3.000 & 0.672 \\
Path C & 5 & $1 / 3$ & 1 & 1.186 & 0.265 \\
\hline
\end{tabular}

Table 7. Evaluation Score of Alternatives.

\begin{tabular}{lcccc}
\hline & Safety & Steepness & Crowded & Bumpiness \\
\hline Path A & 0.552 & 0.072 & 0.110 & 0.063 \\
Path B & 0.096 & 0.279 & 0.076 & 0.672 \\
Path C & 0.352 & 0.649 & 0.814 & 0.265 \\
\hline
\end{tabular}

\section{Results}

The normalized weights of the compared evaluation factors shown in Table 7 are plotted against the values of 'Accountable Degree' shown in gray color in Table 2. The boundary region for each of the alternatives from Lower Model (called L-Evaluation) and Upper Models (called U-Evaluation) are calculated. The L-Evaluation is used for a 'defensive' approach, whereas the U-Evaluation is used for an 'offensive' approach by the robot. 
The L-Evaluation and U-Evaluation plots are shown in in Figures 3 and 4, respectively. The total area under the curve for each alternative is calculated in both the models. In the L-Evaulation, Path-A has an area of 0.348 units, Path-B has an area of 0.087 units, and Path-C has an area of 0.335 units. Similarly, in the U-Evaluation, Path-A has an area of 0.552 units, Path-B has an area of 0.190 units, and Path-C has an area of 0.400 units. In both the models, Path-A scores the maximum area, and therefore, Path-A is selected by the robot to navigate in both defensive and offensive approaches. If Path-A cannot be used (for ex. due to some dynamic obstacle), then Path- $C$ will be selected by the robot to navigate towards its goal.

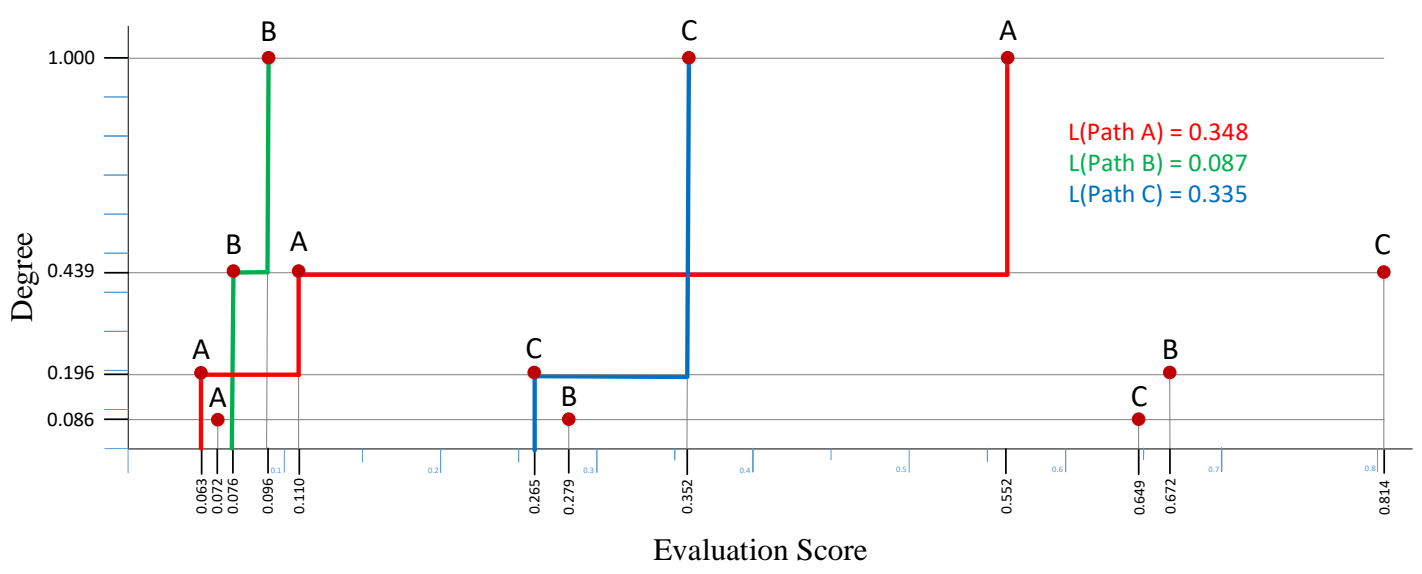

Figure 3. L-Evaluation (Lower Model). Figure is not to scale.

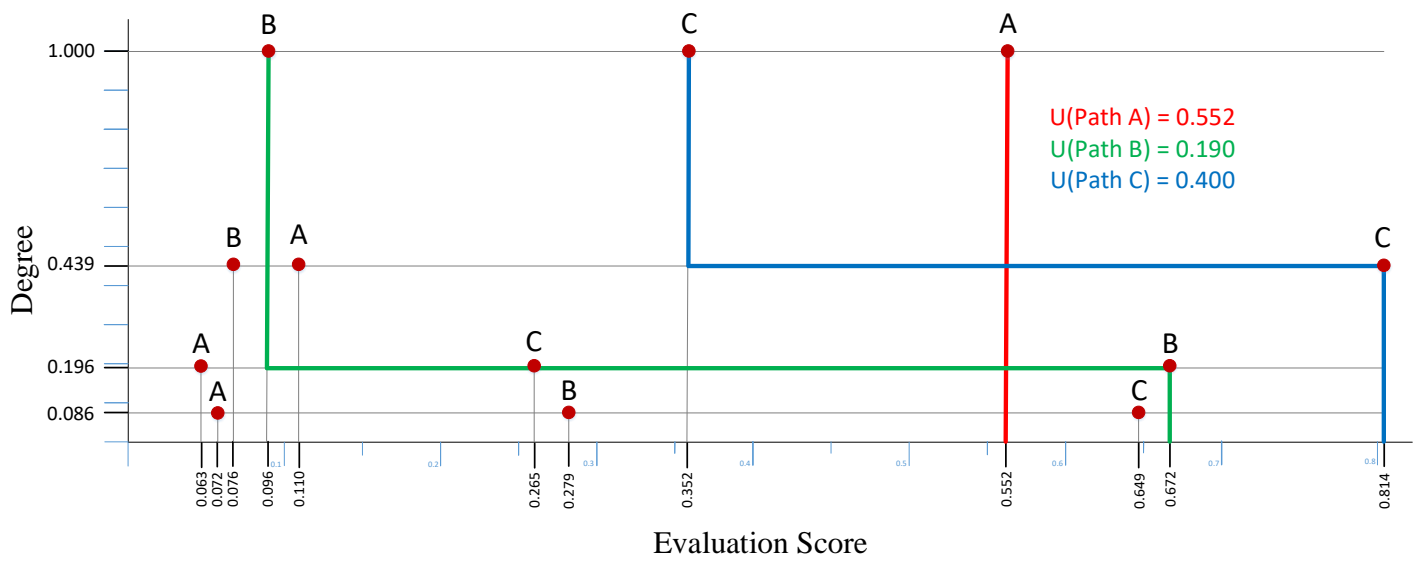

Figure 4. U-Evaluation (Upper Model). Figure is not to scale.

\section{Conclusions}

Mobile service robots are always making some decision or the other and must select the best option from multiple alternatives governed by different factors. In such cases, validating a particular selection based on a rigorous mathematical analysis by quantifying the factors is necessary. Analytical Hierarchical Process can be used in such situations to make the optimal selection. This paper presented a case study of path selection by a robot from several alternative paths based on factors like safety, steepness, crowd, and terrain roughness. It should be noted that AHP does not give a 'correct' decision, but rather helps in decision making by finding the best alternative which best suits the goal by incorporating human judgment in the process. 
Author Contributions: Abhijeet Ravankar and Ankit A. Ravankar conceived the idea, and carried out the experiments for the path planning case study. Yukinori Kobayashi gave valuable suggestions to analyze and improve the results. Takanori Emaru provided the tools.

Conflicts of Interest: The authors declare no conflict of interest.

\section{References}

1. Ravankar, A.; Ravankar, A.A.; Kobayashi, Y.; Emaru, T. SHP: Smooth Hypocycloidal Paths with Collision-Free and Decoupled Multi-Robot Path Planning. Int. J. Adv. Robot. Syst. 2016, 13, 133, doi:10.5772/63458.

2. Ravankar, A.; Ravankar, A.; Kobayashi, Y.; Emaru, T. Intelligent Robot Guidance in Fixed External Camera Network for Navigation in Crowded and Narrow Passages. Proceedings 2016, 1, 37, doi:10.3390/ecsa-3-D008.

3. Ravankar, A.; Ravankar, A.A.; Hoshino, Y.; Emaru, T.; Kobayashi, Y. On a Hopping-points SVD and Hough Transform Based Line Detection Algorithm for Robot Localization and Mapping. Int. J. Adv. Robot. Syst. 2016, 13, 98, doi:10.5772/63540.

4. Saaty, T.L. Decision making with the analytic hierarchy process. Int. J. Serv. Sci. 2008, 1, 83-98.

5. Kim, C.; Langari, R. Analytical Hierarchy Process and Brain Limbic System combined strategy for mobile robot navigation. In Proceedings of the 2010 IEEE/ASME International Conference on Advanced Intelligent Mechatronics, Montreal, QC, Canada, 6-9 July 2010; pp. 967-972.

6. Chan, C.C.; Yu, S.R. Analytic hierarchy process and Zhou-Yi tagging method for knowledge map navigation. In Proceedings of the 2010 IEEE 17Th International Conference on Industrial Engineering and Engineering Management, Xiamen, China, 29-31 October 2010; pp. 1652-1656.

7. Ishizaka, A.; Labib, A. Review of the main developments in the analytic hierarchy process. Expert Syst. Appl. 2011, 38, $14336-14345$.

8. Alam, S.S.; Shrabonti, G. Ranking by AHP: A rough approach. In Proceedings of the Fifth International Conference on Information Fusion, Annapolis, MD, USA, 8-11 July 2002; IEEE Cat.No.02EX5997; Volume 1, pp. 185-190.

9. Ho, W. Integrated analytic hierarchy process and its applications-A literature review. Eur. J. Oper. Res. 2008, 186, $211-228$.

10. Wikipedia. Analytic Hierarchy Process. 2017. Available online: https://en.wikipedia.org/wiki/Analytic_ hierarchy_process (accessed on 5 November 2017).

11. Ravankar, A.A.; Hoshino, Y.; Ravankar, A.; Jixin, L.; Emaru, T.; Kobayashi, Y. Algorithms and a Framework for Indoor Robot Mapping in a Noisy Environment using Clustering in Spatial and Hough Domains. Int. J. Adv. Robot. Syst. 2015, 12, 27.

12. Ravankar, A.; Ravankar, A.A.; Kobayashi, Y.; Jixin, L.; Emaru, T.; Hoshino, Y. An intelligent docking station manager for multiple mobile service robots. In Proceedings of the 2015 15th International Conference on Control, Automation and Systems (ICCAS), Busan, Korea, 13-16 October 2015; pp. 72-78.

(C) 2018 by the authors. Licensee MDPI, Basel, Switzerland. This article is an open access article distributed under the terms and conditions of the Creative Commons Attribution (CC BY) license (http:/ / creativecommons.org/licenses/by/4.0/). 